\title{
Modeling, Measuring, and Compensating Color Weak Vision
}

\author{
Satoshi Oshima, Rika Mochizuki, Reiner Lenz and Jinhui Chao
}

\section{Linköping University Post Print}

\section{Tweet}

N.B.: When citing this work, cite the original article.

(C)2016 IEEE. Personal use of this material is permitted. However, permission to reprint/republish this material for advertising or promotional purposes or for creating new collective works for resale or redistribution to servers or lists, or to reuse any copyrighted component of this work in other works must be obtained from the IEEE.

Satoshi Oshima, Rika Mochizuki, Reiner Lenz and Jinhui Chao, Modeling, Measuring, and Compensating Color Weak Vision, 2016, IEEE Transactions on Image Processing, (25), 6, 2587-2600.

http://dx.doi.org/10.1109/TIP.2016.2539679

Postprint available at: Linköping University Electronic Press

http://urn.kb.se/resolve?urn=urn:nbn:se:liu:diva-128718 


\title{
Modelling, Measuring and Compensating Color Weak Vision
}

\author{
Satoshi Oshima, Rica Mochizuki, Reiner Lenz, Jinhui Chao
}

\begin{abstract}
We use methods from Riemann geometry to investigate transformations between the color spaces of color-normal and color weak observers. The two main applications are the simulation of the perception of a color weak observer for a color normal observer and the compensation of color images in a way that a color weak observer has approximately the same perception as a color normal observer. The metrics in the color spaces of interest are characterized with the help of ellipsoids defined by the just-noticable-differences between color which are measured with the help of color-matching experiments. The constructed mappings are isometries of Riemann spaces that preserve the perceived color-differences for both observers. Among the two approaches to build such an isometry, we introduce normal coordinates in Riemann spaces as a tool to construct a global color-weak compensation map. Compared to previously used methods this method is free from approximation errors due to local linearizations and it avoids the problem of shifting locations of the origin of the local coordinate system. We analyse the variations of the Riemann metrics for different observers obtained from new color matching experiments and describe three variations of the basic method. The performance of the methods is evaluated with the help of semantic differential (SD) tests.
\end{abstract}

Index Terms-Color vision, color weak, color transformations, Riemannian geometry, Riemann normal coordinates

\section{INTRODUCTION}

As many as eight percent of the male population and 0.4 percent of women are color blind and even more suffer some kind of color vision defects in various degrees. We will refer to these less severe color vision defects as colorweakness. Apart from these cases of deficient color vision it is also obvious that there are significant variations of the color vision properties of persons with normal color vision. In critical applications, such as the design traffic signals or natural disaster alarm, one has to make sure that even color blind persons can easily recognize these symbols. Very often this is achieved by enhancing the color contrast between the symbol and the background. Obviously this is not possible for natural images.

Based on this description of color-vision related problems one could formulate the ultimate goal of an adaptive color presentation between two types of observers as follows: Given a stimulus $S$, generate a modified stimulus $S^{\prime}$ such that the perception of observers of type one viewing $S$ is the same as the perception of observers type two viewing $S^{\prime}$. Two

S. Oshima is with Dept. of Information and System Engineering, Chuo University, Tokyo, Japan.

R. Mochizuki is with NTT Service Evolution Laboratories, Tokyo, Japan.

R. Lenz is with Dept. Science and Technology and the Dept. Electrical Engineering Linköping University, SE-60174 Norrköping, Sweden.

J. Chao is with Dept. of Information and System Engineering, Chuo University, Tokyo, Japan. examples would be methods to simulate the perception of color weak or color blind observers and methods to compensate the effects of color weak deficiencies.

In this generality the problem cannot be solved, simply because it is impossible to compare color perceptions of different observers objectively. In fact, even on the sensor-related, low-level we cannot measure quantitative properties of human perception with objective and non-invasive methods and it is therefore impossible to characterize the color-weakness of an observer exactly.

One approach that avoids these problems is the usage of color matching based methods which characterizes the properties of the color perception of an observer by measuring local color differences (see [1], [2]). In this paper we develop methods that modify the input colors in such a way that resulting subjective color differences for two observers are as similar as possible. Geometrically this means that we match color discrimination thresholds of the color-weak and the colornormal observer. These small color differences or discrimination thresholds (typical examples are the MacAdam ellipsoids) are one of the fundamental color vision characteristics and among the few observables in color perception which can be used to characterize the perceptual characteristics of individual observers. Such a discrimination ellipsoid describes the justnoticeable difference (jnd) between the color at the center of the ellipsoid and other colors in its neighborhood. The applied methods were developed in the framework of Riemann geometry. In this framework these ellipsoids characterize the local geometry around center points, and the color space becomes a Riemann space with the thresholds defining the Riemann metric tensor.

Measuring color is of course necessary in many different applications and not only in psychophysics. It is therefore not surprising that there are a large number of different methods to do so and each of these measures defines in turn a color space. Historically there are a large number of such color spaces (see [3] for a historical overview) and the spaces based on Riemann geometry form a major type of such color spaces (see [4], Chap. 8.4). The main advantage of these models is that the locally varying properties of color differences are in direct correspondence in the spatially varying metric of the mathematical space. We also point out that we make no assumptions about the underlying mechanisms that lead to different color perception properties. Relevant factors are possible differences in the spectral absorption properties of the cones, the spatial distribution and relative frequencies of the cone-types or differences in the later color processing steps in the visual system. These factors are all important and for a more details about the background, newer results and 
applications to visualizations we refer to [4], [5] and [6]. We also mention that similar problems are also studied in biology. As an example we refer the interested reader to [7] where experiments studying the color discrimination of chicken (and human observers) are described. This is interesting since birds are believed to have some of the best vision on the planet. The authors also discuss to what extend physics-based models can explain the experimental results. Even though these models provide many valuable insights, it is difficult to find a model fully describing color weakness and individual variations. Identification of the model parameters from measurement data is also very hard and these models are therefore not suitable in the determination of the compensations required for colorweak observers.

A map between color spaces that preserves the local discrimination thresholds everywhere is a local isometry. A global isometry or a compensation map can be obtained by integrating these local mappings using tools from Riemann geometry. Details of a method based on this strategy can be found in [8], [9], [10]. For special types of color weakness, 1-D compensation methods in closed forms and their fast implementation are described in [10] and evaluations of this approach are reported in [11] and [12]. These mappings use techniques from linear algebra and they are therefore easy to implement. However, for many color-weak observers these methods are not general enough and simultaneous compensations in more than one dimension are necessary. There is thus a need for 2D and 3D methods similar to the ones based on these local affine maps. Unfortunately straight forward generalizations have a limited performance due to the approximation errors originating in the local linearization. An additional problem is the estimation of the corresponding positions of the origins of the local neighborhoods.

In this paper we present a compensation without local linearization based on the global Riemann geometry of 3D color spaces that avoids these problems. This approach is based on an intricate relationship in Riemann geometry between a global isometry and a local isometry between two Riemann spaces. The mathematical background behind this approach is the following: The metric tensor defines lengths and angles in the tangent space at a point. This can be used to construct geodesics (corresponding to straight lines) on the manifold. For two points on such a geodesic we can define the distance between these two points as the length of the segment of the geodesic connecting them. The theorem of Myers-Steenrod ([13], page 132) states that a bijective map between two Riemann manifolds is a local isometry if it is a global isometry or distance preserving. It is also true that local isometries are global isometry (page 133). The details and the proof can be found in Chapter 5 of [13]. We will introduce a procedure to construct a global isometry by using multiple Riemann normal coordinates which consist of geodesics based on the local metric in (2D and 3D) color spaces.

The construction of the isometry requires the knowledge of the metric tensor. We acquire the necessary data by measuring color discrimination threshold data for both color-normal and color-weak observers. In [14] discrimination threshold data was measured on the chromaticity plane of the CIEXYZ space and a 2D compensation was implemented. The experiments showed that more accurate threshold data were required and that chromaticity information alone was, in general, not sufficient to produce satisfying compensation results. This showed that a simultaneous compensation of both lightness and chromaticity in $3 \mathrm{D}$ color spaces is needed. Here we describe a new algorithm for fast and stable measurement of the 3D threshold data. As a result we obtain a new set of discrimination threshold measurements for both colorweak and normal observers in CIELUV space of reasonably high resolution. We describe this new dataset and we then discuss the implementation of the color-weak compensation, based on these new measurements, regarding the cost and performance. We consider (in increasing order of complexity) three algorithms: 2D, 2D+1D and 3D compensation maps. This results in global isometries or color-weak maps, that simulate the color-weak vision for a color normal observer. Its inverse can be used as compensation map that transforms a given image as so that the color-weak observer can experience a similar color perception as the corresponding color-normal observer. We apply the method to natural images and evaluate its performance using semantic differential (SD) tests. We use this method to measure the similarity between judgments of different observers. The basic step in this evaluation consists of a presentation of an image to an observer who is asked to rate the properties of the image with the help of a binary scale. The overall ratings of different observers is compared with the help of a correlation coefficient. Details of the procedure are described in Section VIII. The interested reader may consult the standard reference [15] for details and background information.

\section{RIEMANN GEOMETRY OF COLOR SPACES}

An $n$-dimensional Riemann space is a space that locally looks like a Euclidean space $\mathbb{R}^{n}$ with a metric which is defined by an $n$ by $n$ matrix $G$ such that the squared length of a vector $x$ is defined as $x^{T} G x$ where $(\cdot)^{T}$ denotes vector transposition ([4], [16], [13]). In a Riemann space, the local geometry is described by the metric tensor $G(x)$ for every point $x$ in the space where $G(x)$ is a positive-definite symmetric matrix smoothly varying along $x$ defining the local distance around $x$. The matrix $G(x)$ defines a quadratic form on the tangent space at $x$ where the squared length of a tangent vector $d x$ is given by

$$
\|d x\|^{2}=d x^{T} G(x) d x .
$$

Actually, color vision was one of the application areas mentioned by Riemann when he introduced his new geometrical construction and Wright's intervals and MacAdam ellipses and ellipsoids are among the first measurements of the metric tensor in 1D, 2D and 3D spaces (see [4],[17] for a historical overview).

In color science the local metric is estimated with the help of color matching experiments. In such an experiment an observer compares two colors, a fixed test color and a second color. At the beginning of the experiment the two colors are identical. The observer can control the second color and move it a certain direction in a color space until he/she can see 
a difference between the test color and the modified color. This color difference is known as the just-noticeable-difference (jnd) or a color discrimination threshold. A series of such matching experiments, where the secondary color is moved in different directions in color space, can be used to construct the matrix $G(x)$. Details of this construction and a new version will be described later in the experimental part of the paper.

The distance $d\left(x_{1}, x_{2}\right)$ between two points $x_{1}, x_{2}$ in a Riemann space is defined as the length of the shortest path $\gamma_{12}$ connecting the two points. These paths are the geodesics and large color differences in color spaces can be defined is this distance on the Riemann manifold:

$$
d\left(x_{1}, x_{2}\right)=\int_{\gamma_{12}}\|d x\|=\int_{\gamma_{12}} \sqrt{d x^{T} G(x) d x} .
$$

We model the color perception of an individual observer by deriving the metric of the color space from his/her threshold data and subjective color differences are then measured as distances in the obtained color space as a Riemann space. By comparing and preserving the distance between points in the color spaces of two observers we can compare and preserve subjective properties of the color vision of these observers. A map that preserves distances between two Riemann spaces is known in geometry as an isometry. A local isometry is defined as a map between two spaces $C_{1}$ and $C_{2}$ :

$$
f: C_{1} \longrightarrow C_{2}: x \longmapsto y=f(x)
$$

that preserves local distances in the neighborhood of all points. This implies that $f$ maps space $C_{1}$ with Riemann metric $G_{1}(x)$ to space $C_{2}$ with metric $G_{2}(y)$ such that for every $x$ in $C_{1}$

$$
G_{1}(x)=\left(D_{f}\right)^{T} G_{2}(y) D_{f},
$$

where $D_{f}$ is the Jacobian of $f$. Local isometries are functions that map the threshold ellipsoid at every $x$ onto the corresponding threshold ellipsoid at $y=f(x)$. A global isometry is a map $f$ such that the distances $d\left(x_{1}, x_{2}\right)$ for every pair of $x_{1}, x_{2}$ in $C_{1}$ is the same as $d\left(y_{1}, y_{2}\right)$ for $y_{1}=f\left(x_{1}\right), y_{2}=f\left(x_{2}\right)$ in $C_{2}$. It can be shown that a bijective local isometry also gives a global isometry and vice versa [13].

\section{COMPENSATION OF COLOR WEAK VISION WITH ISOMETRIES}

So far we described how color matching experiments provide input data that can be used to construct a Riemann manifold model that characterizes the properties of the low-level part of the color vision system of an observer. In this framework isometries can be constructed that preserve the geometric structure of the color spaces involved. The assumption is that with the help of such a mapping we can generate a visual input that allows us to convey the color perception of one observer to another observer. As a result it should be possible to share the perceptional experience between observers (at least on this low-level of color perception). Constructing color-differencepreserving maps $f$ between observers is then equivalent to the construction of isometries between Riemann manifolds.

As mentioned before, we are mainly interested in the relation between the color perception of color-weak and colornormal observers. We define a color-weak map $w$ as an isometry from the color space $C_{w}$ of the color-weak observer to the space $C_{n}$ of the color-normal observer as

$$
w: C_{w} \longrightarrow C_{n}: y=w(x)
$$

This map shows to the color-normal observers what is actually seen by the color-weak observer and provides thus a color-weak simulation map, which simulates the color-weak perception from a color-normal's point of view. The inverse map of the color-weak map $w$ is also an isometry, now from the color space of the color-normal observers to the color space of a color-weak observer

$$
w^{-1}: C_{n} \longrightarrow C_{w}: x=w^{-1}(y)
$$

which shows to the color-weak observer what the color-normal observer actually sees, it is thus a compensation map.

There are two ways to construct isometries: one can either match discrimination threshold ellipsoids by local affine maps or one can build the global isometry between Riemann spaces directly. The method using local affine maps has the advantage to it is easier to understand and easier to implement using basic linear algebra. Descriptions of this approach can be found in [8], [9] and [18]. There are two major problems with this approach: numerical errors due to approximation in linearization are significant and it is difficult to match the positions and the correspondences between the origins of the local neighborhoods in the two spaces. The second approach to compute the isometry directly is also difficult since the proof of the existence of a global isometry gives very few clues regarding the practical implementation. In the following we will introduce a method to build a global isometry which uses Riemann normal coordinates. First attempts to implement this method are reported in [19] and [20].

We will first consider the special case where we make use of the Riemann normal coordinates in a Riemann space to obtain an isometry between the Riemann space and the Euclidean space. We call this an uniformization from the color space $C$ to the Euclidean space $U$ and denote it by $p$. Now, instead of constructing the isometry between color spaces $C_{i}, i=1,2$ directly we will construct first uniformizations $p_{i}$ to the common Euclidean space $U$. Since the inverse of an isometry and composition of two isometries are also isometries, we obtain the isometry between $C_{1}$ and $C_{2}$ as $f=p_{2}^{-1} \circ p_{1}$. The Riemann normal coordinates can be regarded as a generalization of the polar coordinates in the Euclidean space and we will construct the isometric image of the polar coordinates in the Euclidean space.

\section{Construction OF ISOMETRIES By RiemanN NORMAL COORDINATES}

In Eq.(2) we introduced the distance between two colors as the length of the geodesic connecting the two points. A geodesic $u$ in a Riemann space corresponds to a straight line in a Euclidean space. It can be shown [16] that it is given by the solution of the differential equations (in the local coordinates $u^{i}$ )

$$
\frac{d^{2} u^{i}}{d s}+\Gamma_{j k}^{i} \frac{d u^{j}}{d s} \frac{d u^{k}}{d s}=0
$$




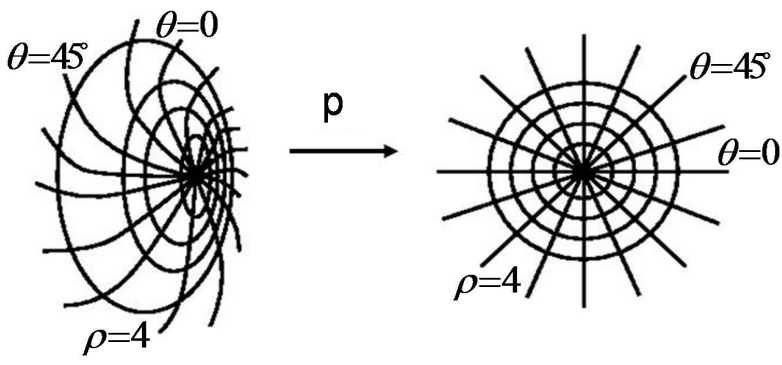

Fig. 1: Uniformization of a color space using Riemann normal coordinates

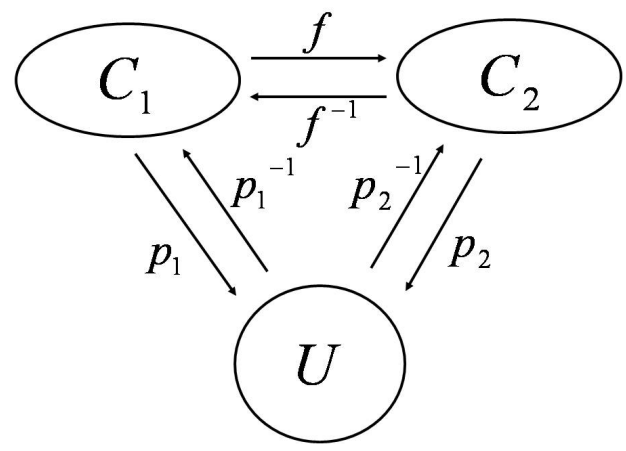

Fig. 2: Construction of global isometry between two arbitrary spaces

where $\Gamma_{j k}^{i}$ is the Christoffel symbol defined as:

$$
\Gamma_{j k}^{i}=\frac{1}{2} g^{i \alpha}\left(\frac{\partial g_{\alpha j}}{\partial u^{k}}+\frac{\partial g_{\alpha k}}{\partial u^{j}}-\frac{g_{j k}}{\partial u^{\alpha}}\right) .
$$

As usual the metric tensor and its inverse are denoted by

$$
G=\left(g_{i j}\right), G^{-1}=\left(g^{i j}\right)
$$

and the Einstein summation convention is used.

Such a system can be solved by choosing an origin and the initial conditions e.g. the speed at the origin. The origin can be chosen as certain reference point which does not change between color normal and color weak observer. Natural choices in color science are the points specified by the white points D65 or D50.

The initial conditions for the geodesics eminating from the origin could include the requirement of a unit length speed vector (therefore the natural parameter or curve length is used for parameterization) and the condition that the geodesics should be separated in the equal angles (lengths of curves and the angles between them are measured using Riemann metric $G(x)$ at the origin). However, these conditions are not sufficient to uniquely specify the coordinates systems in two different spaces to have the same reference direction. In the $2 \mathrm{D}$ case we have to ensure that the directions of the zero degree geodesics are identical. In the $3 \mathrm{D}$ case, it is even more complicated since the Frenet formula for spatial curves states that one needs to specify both the direction of the tangent vector of the geodesic and a second vector either the acceleration or the torsion. In the following we use the invariant chroma in [21] as the common reference directions for both color normal and color weak observers. In our case this is the lightness direction and e.g. the blue ( $\mathrm{S}$ cone excitation) for the color-normal and color-weak observers of type $P$ and $D$ since for them the perception of blue is the same.

The geodesics can be used to build a coordinate system as follows: every point lies on one geodesic and we can thus describe the location of the point by first specifying on which geodesics it lies and then use its distance to the origin. Points with the same distance from the origin form a circle or sphere centered at the origin. In the current application such a circle/sphere defines an equi-chroma surface. In our implementation we calculate the Riemann normal coordinates of a given point by first finding the cell in the coordinates grid which contains the point, then the distance and angle of the point is determined e.g. by convex interpolation in the cell. The procedure to map the original coordinates to the Riemann normal coordinates can be applied to build Riemann normal coordinates of multiple neighborhoods. The global coordinates can be obtained using standard coordinate transformation from local coordinates.

\section{MEASUREMENT OF DISCRIMINATION THRESHOLD DATA}

Classical psychophysical measurement methods are the method of limits, the method of adjustment and the method of constant stimuli (see [1] for a description). Using the method of limits the experimenter presents stimuli stepwise in ascending or descending order and notes when the observer detects a change. In the method of adjustments the experimenter or the observer adjusts the strength of the stimuli in a continuous manner until the observer notices the change. In the method of constant stimuli the experimenter presents a number of stimuli in random order and notices the reactions of the observer.

In order to achieve a correct measurement, it is necessary to avoid bias due to anticipation, adaptation or learning effects and fatigue of observers during the experiments. The totally random measurement method is the most effective procedure avoiding these biases and therefore the most precise. However, unlike the method of limits and the method of adjustments, which make use of the preceding results of measurement to trace a certain paths among all combinations of the test colors, the random measurement approach needs to present all combinations of the test colors, therefore, it is also the most time consuming data collection method. In our experiments we use a randomized adjustment method in pair-wise comparison experiments to determine the color discrimination thresholds. A session of color-matching starts with the display of the test color on the left and a comparison color on the right. The observer is asked to use either the mouse wheel or a keyboard to adjust the comparison color until it matches the test color as close as possible. An accepted match finishes the session.

For a given test color the comparison color is randomly chosen on straight lines in 14 directions centered at the test color. These 14 directions consist of the 6 directions along 
coordinate axes and the eight directions connecting the center point to the eight corners of the unit cube. The initial position of the comparison color and the increment or decrement along each direction are randomly chosen from certain ranges and/or a selection of discrete values. Thus the observer only decides if the next comparison color is more similar to the test color but the observer has no control over the magnitude of the change of the comparison color. The switching time to the new comparison color needs to be adjusted according to the movement speed of the mouse wheel or the rate of keyboard inputs. We also introduce random variations to this adjustment. We used a SyncMaster XL24 monitor by Samsung illuminated by Panasonic Hf PREMIER fluorescent lamps, a Munsell N5.5 background and a 10 degree viewing angle. The distance between the observer and the screen is $80 \mathrm{~cm}$, the size of the two test and the comparison color frames on the display is $14 \times 14 \mathrm{~cm}$. In our experiments we measured color discrimination thresholds of a normal observer and a typical color-weak deuteranomalous observer (D..type, green-weak). We applied the randomized adjustment method in CIELUV sRGB space. We measured 77 sampling points, at 5 different lightness levels $L^{*}=30,40,50,60,70$. Data grids in the chromaticity planes of these levels contain 9, 13, 19, 20 and 16 points. An ellipsoid at a point is estimated from deviations in 14 directions from the origin.

Step 1 In the pre-adaptation period of 5 minutes before the measurement, a neutral gray (Munsell N5:5) is shown on the whole display.

Step 2 The two $14 \times 14 \mathrm{~cm}$ frames with the test and comparison colors are shown on the display (10 degree vision field, $80 \mathrm{~cm}$ distance, test color on the left, comparison color on the right). The test color is fixed during one session.

Step 3 The observer uses a mouse wheel or keyboard keys which change the CIELUV values of the comparison color based on the algorithm described above.

Step 4 Repeating Step 3, the observer tries to make the comparison color as similar as possible to the test color.

Step 5 The above measurement will be repeated four times. The average value of these results will be used as discrimination threshold data.

Step 6 In a rest period of seven seconds between every four sessions a neutral gray N5:5 is shown on the whole display.

Step 7 The procedure from Step 2 to Step 6 are repeated for all test colors in the color space. This is repeated for at most 90 minutes after which the measuring session ends. The next session will continue no sooner than three days from the end of the current session.

The measurements are repeated four times for each test color to obtain a statistically stable estimate. The ellipsoids are then estimated from the observation data using the methods described in [8], [22] and [23]. Discrimination threshold ellipsoids measured in 3D are shown in Fig. 3 for a color normal observer and Fig. 4. It can be seen that the discrimination threshold ellipsoids differ greatly in size and direction. Table I shows the volume ratio between the ellipsoids of a colornormal observer and a color-weak observer. It can be seen that the discrimination ellipsoids of a color-normal observer are on average 2.7 times larger than those of a color-weak observer. This explains the reduced color vision capabilities of the color-weak observer. One can also observe the differences of the shapes, sizes and orientations of the threshold ellipsoids between different lightness levels.

TABLE I: Volume ratio of the ellipsoids between color-normal and color-weak observers

\begin{tabular}{c|c}
$(L *)$ & Average volume ratio \\
\hline 70 & 3.1989 \\
60 & 1.7918 \\
50 & 3.2293 \\
40 & 1.2089 \\
30 & 4.8231 \\
Overall & 2.6948
\end{tabular}

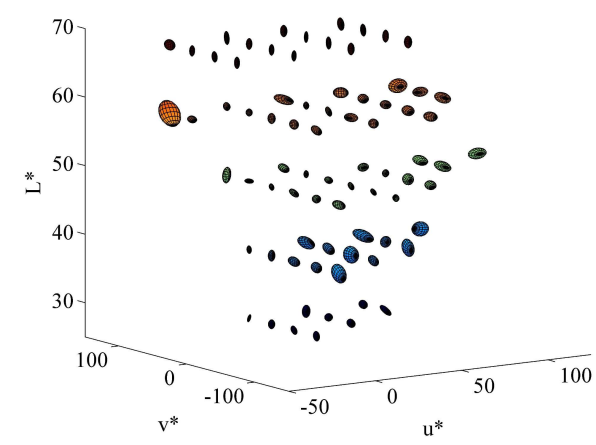

Fig. 3: Discrimination ellipsoids of a color-normal observer

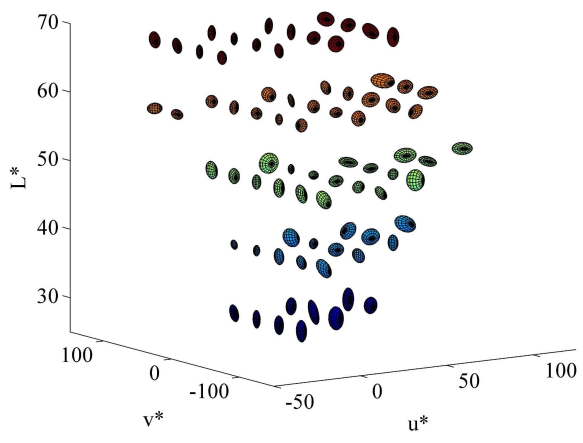

Fig. 4: Discrimination ellipsoids of a D-Type color-weak observer

Colors that are not discriminated by an observer with color deficiency of a single cone are located on a line, the so-called confusion line of the type of color-weakness (see [4]). In Figures 5, 6, 7 and 8 we show the discrimination ellipses for the $L^{*}=30,40,50$ and $L^{*}=60$ lightness planes of a color-normal observer and the D-type color-weak observer. The frame indicates the gamut at this lightness level while the 


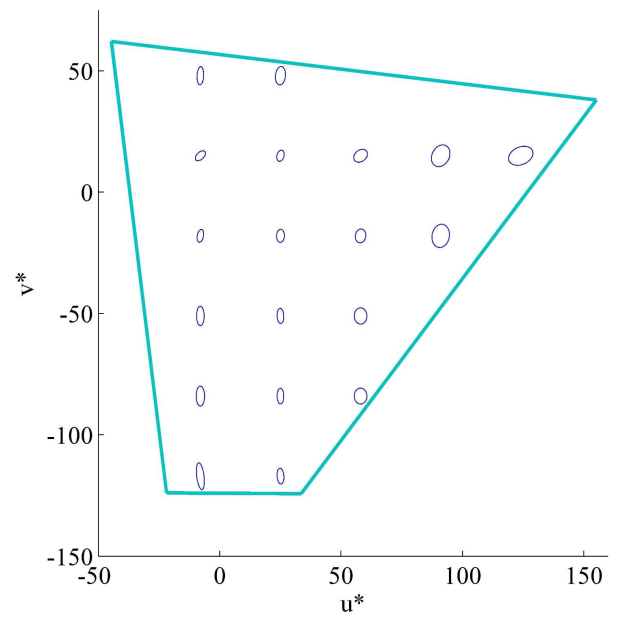

Fig. 5: Discrimination ellipses of a color-normal observer $\left(L^{*}=\right.$ 50)

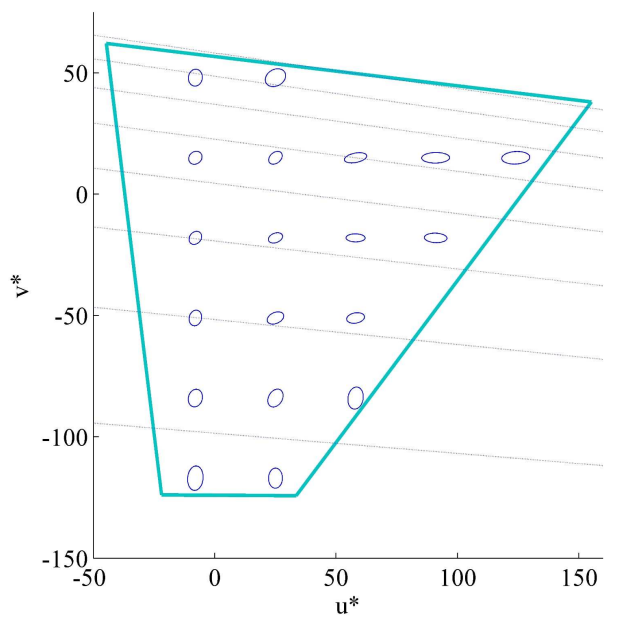

Fig. 6: Discrimination ellipses of a $D$-Type color-weak observer $\left(L^{*}=50\right)$

TABLE II: Area ratio of the ellipsoids between color-normal and color-weak observers

\begin{tabular}{c|c}
$L^{*}$ & Area ratio of ellipses \\
\hline 70 & 2.5172 \\
60 & 1.2074 \\
50 & 1.5590 \\
40 & 0.9675 \\
30 & 2.0069 \\
Overall & 1.6193
\end{tabular}

dashed lines show the confusion lines the D-type color-weak observers. The differences between discrimination thresholds of the color-normal and the color-weak observers can be clearly seen. In Table II we collect the area ratio between the discrimination ellipses of the color-normal and the color-weak observers. The values are almost all greater than one which was to be expected.

At the same time, it is interesting to notice that although

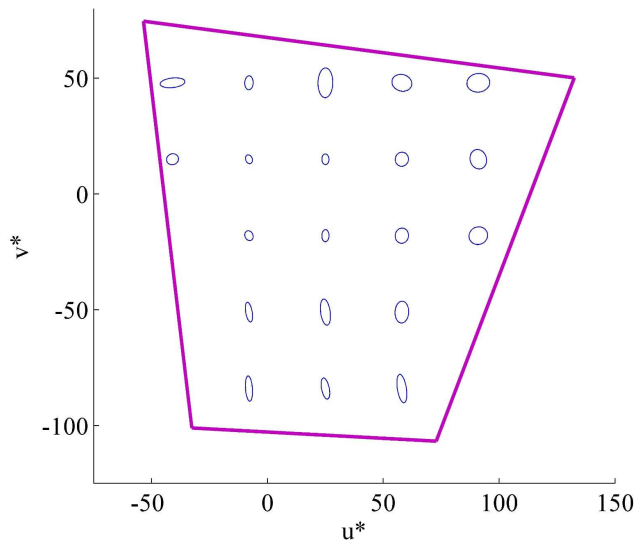

Fig. 7: Discrimination ellipses of a color-normal observer $\left(L^{*}=\right.$ $60)$

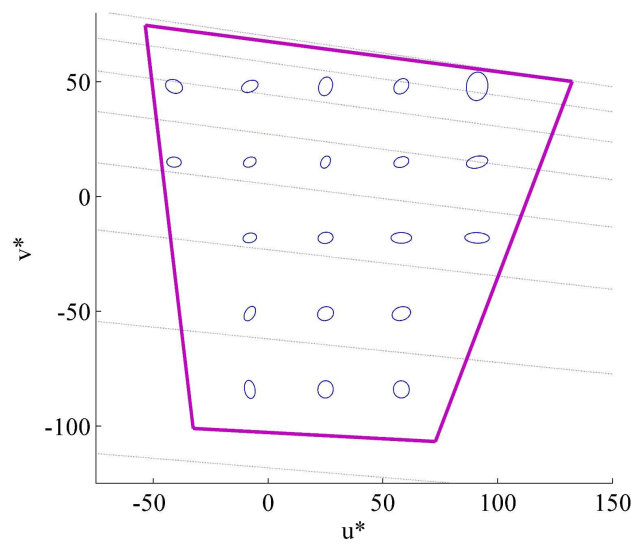

Fig. 8: Discrimination ellipses of a $D$-Type color-weak observer $\left(L^{*}=60\right)$

the color-weak observer is diagnosed by the Anomaloscope as D-type, the shapes, sizes and orientations of the ellipses vary widely over the color space. In particular, they are not always extended only along the confusion lines of D-type color-weakness or the $M$ direction in the LMS color space which indicates greater insensibility to green for a typical $D$ type observer. The discrimination threshold ellipsoids actually extend simultaneously also along the confusion lines of $P$ Type and $T$-Type color-weakness or the $L^{*}$ and $S$ directions in the LMS color space which suggest greater insensibility to red and blue. In fact, this is often found in discrimination threshold measurements These deviations of the ellipses from the confusion lines of the "diagnosed" color-weak type suggest that usually color weakness occurs in more than one type or direction. Color-weakness is thus rarely of a single type but a compound phenomenon. This also means that it is inefficient to describe color-weakness using 1D models like confusion 
lines. A more precise representation and compensation of color vision characteristics is only possible in higher dimensions. Similarly, one can find that the discrimination threshold ellipses at different lightness levels are also quite different. This can be seen by comparing the results for the levels $L^{*}=50$ and $L^{*}=60$. These results show that color-weakness is a complex deformation of $3 \mathrm{D}$ color space, not only along confussion lines and not only restricted to the chromaticity plane. Practically this also implies that it cannot be exactly described and compensated by a Cartesian product of an 1D model of lightness and a 2D model of the chromaticity plane [24].

\section{CONSTRUCTION OF RIEMANN NORMAL COORDINATES IN COLOR SPACES}

Our measurements show that color-weakness is characterized by a complicated deformation of $3 \mathrm{D}$ color space. Compensation maps and simulation methods should therefore also use three-dimensional geometry. However, the costs of constructing Riemann normal coordinates in higher dimesional spaces is also high and there is thus a trade-off between accuracy and computational costs. In the following we will describe different approximation methods and study their performance. We will describe methods using only chromaticity, approximations based on the Cartesian product of a onedimensional model for lightness and a 2D model for chromaticity and full three-dimensional models. For interpolation of the discrimination thresholds, after a Gaussian smoothing, the cubic B-spline and the Akima algorithm [25] were used to calculate the Christoffel symbols.

An algorithm to build isometries and compensation algorithm in one-dimensional spaces is described in [10]. It was applied to confusion lines for one-dimensional color-weak compensation. Here we use it in the lightness direction to obtain a compensation along the lightness axis. To obtain the Riemann metric on the $L$ axis, we calculate the intersection of the discrimination ellipsoids centered $l$ on the $L^{*}$ axis and the $L^{*}$ axis. The resulting interval on the $L^{*}$-axis defines the jnd threshold on the $L^{*}$ axis. In this way, one obtains the metric tensor $G_{n}(l)$ for the color-normal and $G_{w}(l)$ for the color-weak observers.We then apply the algorithm from [10] to match these metrics in the two spaces to obtain a correspondence between the lightness axes of the colornormal and the color-weak observers. This correspondence defines the isometry or color-weak map between the two onedimensional spaces, which will later be used to build a 2D+1D compensation scheme.

In the case of the two-dimensional chromaticity plane one starts the geodesics from the common origin $\left(u^{*}, v^{*}\right)=(0,0)$ for each lightness level, which is the same for both colornormal and color-weak observer. The common reference direction given by the zero angle in both, the color spaces of the color-normal and the color-weak observers, is defined as the invariant hue given by the mono-chromatic color with $475 \mathrm{~nm}$ [21].

The next step is to calculate geodesics emanating from the origin, uniformly separated in equal angular increments. This is done using the fourth degree Runge-Kutta algorithm. By choosing the unit length tangent vector at the origin we obtain the natural parametrization of the geodesics by their curve lengths. The lengths and the angles are measured using metric tensor at the origin. We computed 36 geodesics from the origin separated by 10 degrees from each other. We also applied the multi-patch strategy here. We thus built an additional coordinate system starting from a second origin which was chosen from a part with low density in the first grid.

The Riemann normal coordinates for normal and color-weak observers are shown in Figs. 9 and 10 for $L^{*}=50$ and in Figs. 11 and 12 for $L^{*}=60$. The frames in the figures indicate the gamut at this lightness level. The blue solid lines are geodesics, the yellow-greenish solid lines are equa-chroma lines. Comparing the coordinates at different lightness levels also revealed a distortion along the lightness direction so that the coordinates system is not a Cartesian product between a $1 \mathrm{D}$ coordinates and a 2D coordinates.

We counted the number of the grid points inside the gamut at each lightness level which describe the size of the gamut. The ratios between the number of grid points of the color-weak over the corresponding number of the color-normal observer are shown in the Table III. For the color-weak observer this ratio is around 0.7918 times of that of the color-normal. The grid points in the two color space are separated by an equal distance due to the isometry and we conclude therefore that the color-weak observer has more sparse coordinate grid than the color-normal observer. This agrees with the previous observations about the difference between the two color perception properties.

TABLE III: Ratio of the number of grid points of color-weak over color-normal observers (2D)

\begin{tabular}{c|c}
$L^{*}$ & Ratio of the number of grid points \\
\hline 30 & 0.7084 \\
40 & 0.9411 \\
50 & 0.7930 \\
60 & 0.8649 \\
70 & 0.6519 \\
Overall & 0.7918
\end{tabular}

In the case of the three-dimensional model the common origin for the Riemann normal coordinates can be chosen as the origin on the lowest level of lightness. e.g. in our case, we selected $\left(L^{*}, u^{*}, v^{*}\right)=(30,0,0)$. In this case we computed $13 \times 18=234$ geodesics emanating from the origin, separated by the same spatial angle and unit initial speed. Distances and angles are again calculated using the metric tensor $G(x)$ at the origin, the common reference direction between the color spaces of color-normal and color-weak observers is again defined by the invariant hue $475 \mathrm{~nm}$ in [21]. We also used the multi-patch algorithm in both color spaces.

The Riemann normal coordinates in CIELUV space of the color-normal and the color-weak observers are shown in Figs 13 and 14 . For better visibility, only geodesics are shown here. The ratio of the number of the grid points inside the gamut for the color-weak over the color-normal observer is 0.6785 , so the difference in the densities of the coordinate grid between 


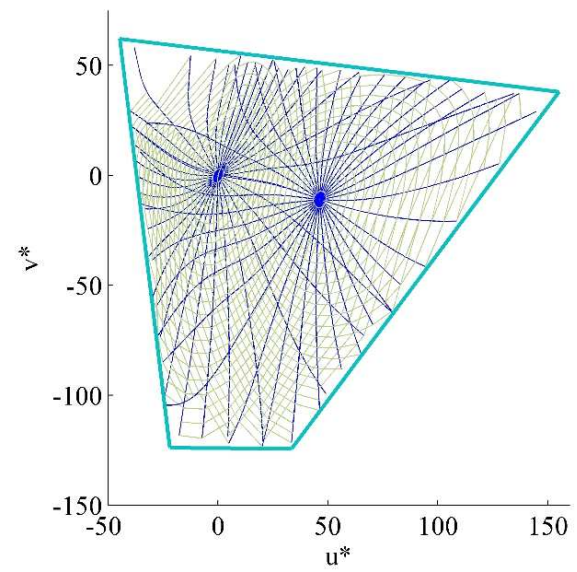

Fig. 9: Riemann normal coordinates for a color-normal observer $\left(L^{*}=50\right)$

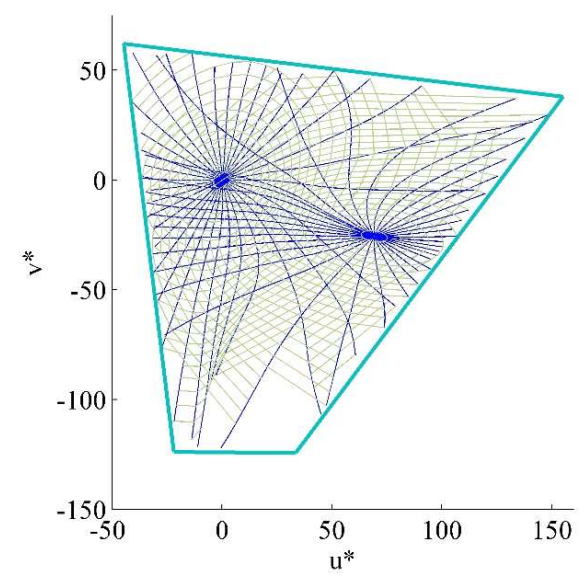

Fig. 10: Riemann normal coordinates for a $D$-type color-weak observer $\left(L^{*}=50\right)$

the color-normal and the color-weak observers is even greater in the $3 \mathrm{D}$ space.

\section{COMPENSATION OF NATURAL IMAGES}

Using the Riemann normal coordinates in the color spaces of the color-normal and the color-weak observers, one can now construct an isometry between two color spaces using the approach described previously. We will now describe the three implementations of the color-weak compensation for the $2 D$, the $2 D+1 D$ and the $3 D$ case.

\section{A. $2 D$ case}

Step 1 For the chromaticity planes with fixed lightness level $l$ in the color-normal and color-weak observers, build the compensation map $\gamma_{l}: C_{n} \longrightarrow C_{w}$ as the inverse map of the color-weak map $w_{l}$ for each $l$;

Step 2 Transform the $R G B$ coordinates of an input image to CIELUV coordinates in $C_{n}$;

Step 3 Choose the $l$ which is the closest to $L^{*}$, and choose the compensation map as $\gamma_{l}$;

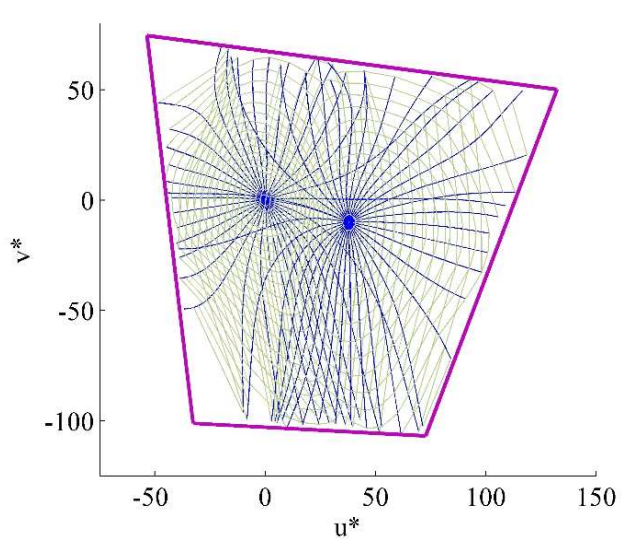

Fig. 11: Riemann normal coordinates for a color-normal observer $\left(L^{*}=60\right)$

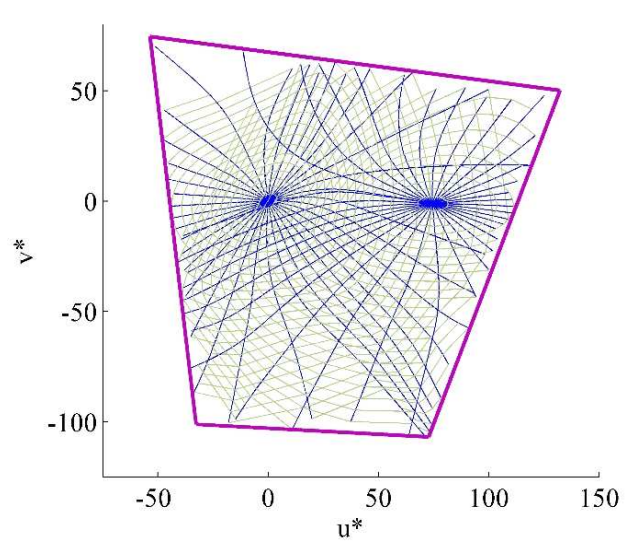

Fig. 12: Riemann normal coordinates for a $D$-type color-weak observer $\left(L^{*}=60\right)$

Step 4 From the grid of the Riemann normal coordinates of $C_{n}$, find the triangular patch which contains $\left(u^{*}, v^{*}\right)$. The Riemann normal coordinates of the input color is taken as e.g. either that of the closest vertex $\left(u_{0}^{*}, v_{0}^{*}\right)$, or a convex combination of the vertices of the triangel $\left(u_{i}^{*}, v_{i}^{*}\right), i=1, \ldots, 3$, i.e.,

$$
\left(u^{*}, v^{*}\right)=\sum_{i} a_{i}\left(u_{i}^{*}, v_{i}^{*}\right), a_{i} \geq 0, \sum_{i} a_{i}=1
$$

Step 5 Apply the map $\gamma_{l}$ to the input color, e.g., the vertex $\left(u_{0}^{*}, v_{0}^{*}\right)$ in $C_{n}$ becomes the vertex $\left(u_{0}^{*^{\prime}}, v_{0}^{*^{\prime}}\right)$ in $C_{w}$, or, assuming that $\gamma_{l}$ is a linear map of the simplex, then the vertices $\left(u_{i}^{*}, v_{i}^{*}\right), i=1, \ldots, 3$ become $\left(u_{i}^{*^{\prime},} v_{i}^{*^{\prime}}\right), i=1, \ldots, 3$, and we get:

$$
\begin{aligned}
\left(u^{*^{\prime}}, v^{*^{\prime}}\right) & =\gamma_{l}\left(\left(u^{*}, v^{*}\right)\right) \\
& =\sum_{i} a_{i} \gamma_{l}\left(\left(u_{i}^{*}, v_{i}^{*}\right)\right) \\
& =\sum_{i} a_{i}\left(u_{i}^{*^{\prime},} v_{i}^{*^{\prime}}\right)
\end{aligned}
$$




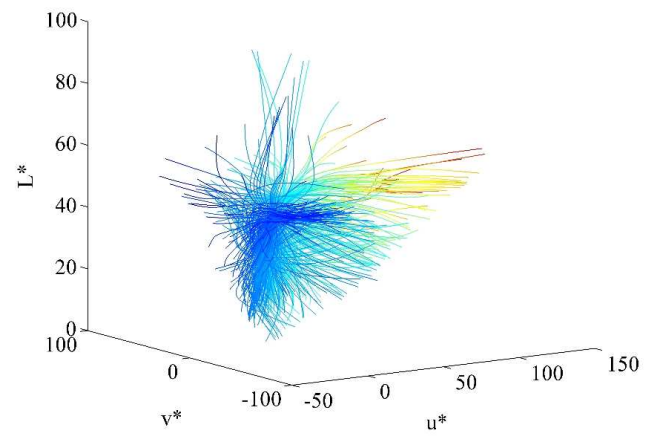

Fig. 13: Riemann normal coordinates in CIELUV space of the color-normal observer

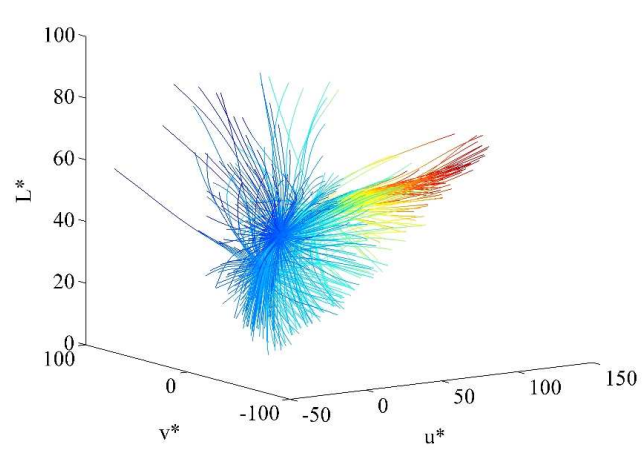

Fig. 14: Riemann normal coordinates in CIELUV space of the $D$-type color-weak observer

Step 6 Repeat Step 3 to Step 6 for all pixels in the input image;

Step 7 Transform the CIELUV coordinates to $R^{\prime} G^{\prime} B^{\prime}$ coordinates

\section{B. $2 D+1 D$ case}

The $2 \mathrm{D}$ algorithm does not take into account the distortion of color-weak vision along the lightness direction. Next we add to the $2 \mathrm{D}$ algorithm a $1 \mathrm{D}$ compensation along the lightness direction. The 1D compensation algorithm described in the previous section is then applied to the $L^{*}$ axis. The algorithm is then exactly the same as in the previous section but with a new step to compensate the $l$ values simultaneously.

\section{C. $3 D$ case}

For the full 3D case we get finally the following processing steps:

Step 1 For color spaces $C_{n}, C_{w}$ of the color-normal and color-weak observers, build the compensation map $\gamma: C_{n} \longrightarrow C_{w}$ as the inverse map of the color-weak $\operatorname{map} w$

Step 2 Transform the $R G B$ coordinates of an input image to CIELUV coordinates in $C_{n}$;
Step 3 From the grid of the Riemann normal coordinates of $C_{n}$, find the tetrahedron which contains $\left(L^{*}, u^{*}, v^{*}\right)$. The Riemann normal coordinates of the input color is taken as e.g. either that of the closest vertex $\left(L_{0}^{*}, u_{0}^{*}, v_{0}^{*}\right)$, or a convex combination of the vertices of the tetrahedron $\left(L_{i}^{*}, u_{i}^{*}, v_{i}^{*}\right), i=1, \ldots, 4$, i.e.,

$$
\left(L^{*}, u^{*}, v^{*}\right)=\sum_{i} a_{i}\left(L_{i}^{*}, u_{i}^{*}, v_{i}^{*}\right), a_{i} \geq 0, \sum_{i} a_{i}=1
$$

Step 4 Apply the map $\gamma$ to the input color, e.g., the vertex $\left(L_{0}^{*}, u_{0}^{*}, v_{0}^{*}\right)$ in $C_{n}$ becomes the vertex $\left(L_{0}^{*^{\prime}}, u_{0}^{*^{\prime}}, v_{0}^{*^{\prime}}\right)$ in $C_{w}$, or , assuming that $\gamma$ is linear on the tetrahedron, the vertices $\left(L_{i}^{*}, u_{i}^{*}, v_{i}^{*}\right), i=1, \ldots, 4$ become $\left(L_{i}^{*^{\prime}}, u_{i}^{*^{\prime},} v_{i}^{*^{\prime}}\right), i=1, \ldots, 4$, then

$$
\begin{aligned}
\left(L^{*^{\prime}}, u^{*^{\prime}}, v^{*^{\prime}}\right) & =\gamma\left(\left(L^{*}, u^{*}, v^{*}\right)\right) \\
& =\sum_{i} a_{i} \gamma\left(\left(L_{i}^{*}, u_{i}^{*}, v_{i}^{*}\right)\right) \\
& =\sum_{i} a_{i}\left(L_{i}^{*^{\prime}}, u_{i}^{*^{\prime},} v_{i}^{*^{\prime}}\right)
\end{aligned}
$$

Step 5 Repeat from Step 3 to Step 5 for all pixels in the input image;

Step 6 Transform the CIELUV coordinates to $R^{\prime} G^{\prime} B^{\prime}$ coordinates

\section{EVALUATION}

Since a direct evaluation of the performance of compensation is difficult, especially for natural images with complicated color distributions, we used the semantic differential method [15] to compare visual impression before and after compensation. The SD method is known as an effective tool for qualitative evaluation of subjective impression by asking an subject to judge between two antonyms for different adjectives.

We selected eight pairs of adjectives from the Osgood's original 76 pairs. These pairs are relevant for judging visual impressions and they avoid adaptation related choices such as "natural or not", "friendly or not".

In one SD-evaluation the current compensation image is calculated using one of the three compensation algorithms and shown to the color-normal and color-weak observers in a random order. Two series of images showing images called "Lake" and "Pond", are shown as examples. After each presentation the observer completes the SD questionnaire. The results and the correlation coefficients between SD scores of the color normal observer seeing the original and the colorweak observer seeing the compensated images are shown in Table IV.

In our evaluation the compensation algorithms are applied to ten natural images. The SD scores obtained are shown in the following four cases, "original" and "2D", "2D+1D", "3D" stand for resulting image by $2 \mathrm{D}, 2 \mathrm{D}+1 \mathrm{D}$ and $3 \mathrm{D}$ compensation algorithms, and color-weak "simulation" of the input image is also shown. For the 30 compensation images, 21 resulted in an increment of the correlation coefficients between the color-normal and the color-weak evaluation. This indicates that the compensation leads to a closer similarity between the perception of the color-weak and the normal observer. 
TABLE IV: Correlation coefficient of SD score

\begin{tabular}{l|cccc} 
Image & original & 2D & 2D+1D & 3D \\
\hline "Lake" & 0.4845 & 0.6204 & 0.8518 & 0.7883 \\
"Pond" & -0.5078 & -0.0265 & -0.2136 & 0.1039
\end{tabular}

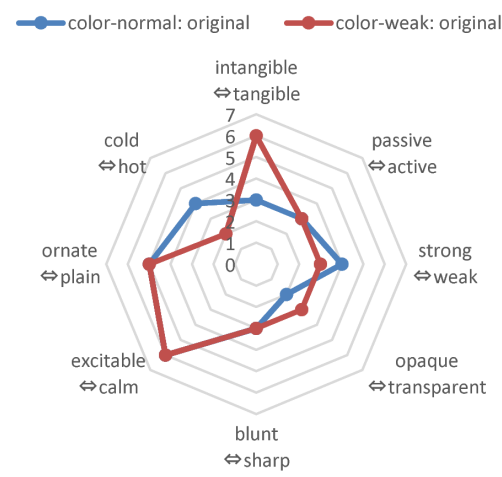

Fig. 15: "Lake":

Color-normal: original D-type color-weak: original

\section{COMPENSATION RESUlts}

The compensation algorithms are applied to ten natural images. The results are shown in the following four cases, "original" and "2D", "2D+1D", "3D" stand for resulting images by $2 \mathrm{D}, 2 \mathrm{D}+1 \mathrm{D}$ and $3 \mathrm{D}$ compensation algorithms, and color-weak "simulation" of the input image is also shown.

In particular, we show two images called "Lake" and "Pond" as the examples.

Table $\mathrm{V}$ shows the area expansion of the pixel distribution on the $u^{*} v^{*}$ chromaticity plane after compensation. The average CIELUV coordinate values are shown in Table VI.

TABLE V: Area expansion of $u^{*} v^{\text {: distribution }}$

\begin{tabular}{l|ll} 
Compensation & "Lake" & "Pond" \\
\hline 2D & 1.8182 & 1.4839 \\
2D+1D & 1.4596 & 1.5483 \\
3D & 1.4017 & 1.4765
\end{tabular}

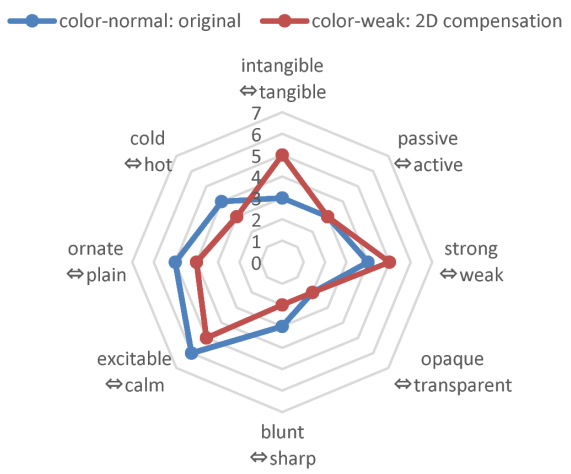

Fig. 16: "Lake"

Color-normal: original D-type color-weak: 2D compensation

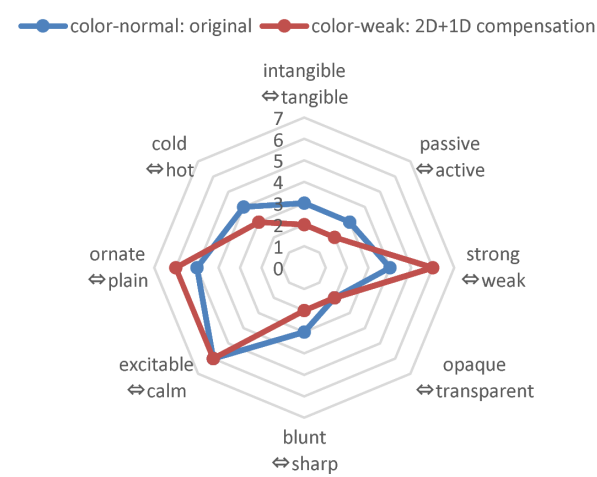

Fig. 17: "Lake":

Color-normal: original D-type color-weak: 2D+1D compensation

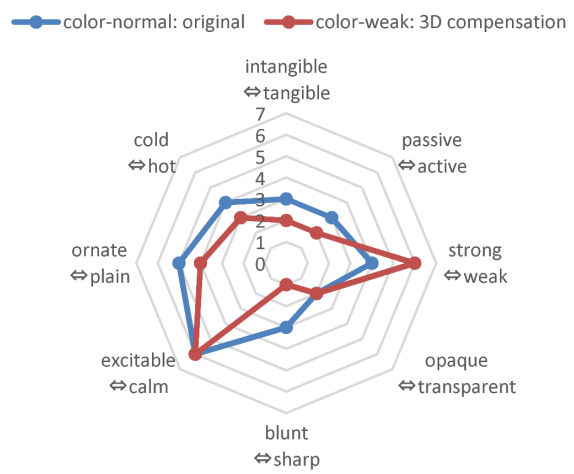

Fig. 18: "Lake":

Color-normal: original D-type color-weak: 3D compensation

Generally the distribution of the pixels in the color space is expanded after compensation. In particular, the chromaticity distribution of the input image has been expanded after compensation. Besides, almost every compensation image has large $u^{*} v^{*}$ average value. After the lightness compensation, the $L^{*}$ average value increased. On the other hand, in the 2D+1D compensation, average of $u^{*}$ and $v^{*}$ decreased, which

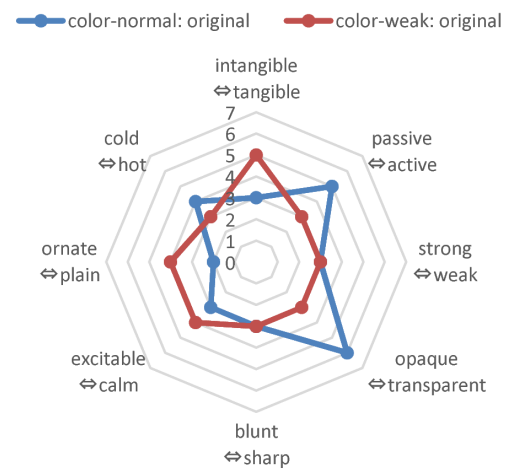

Fig. 19: "Pond":

Color-normal: original D-type color-weak: original 


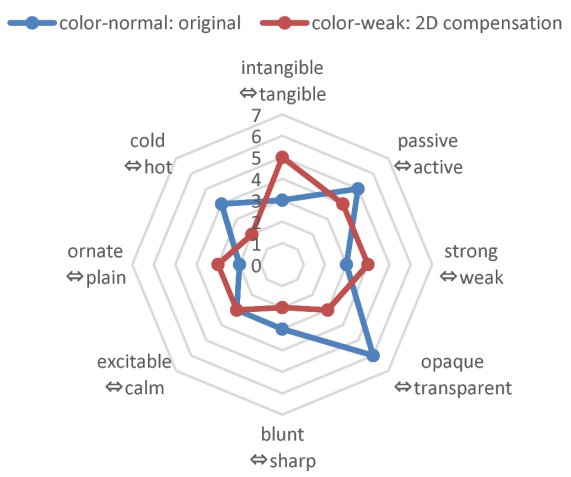

Fig. 20: "Pond":

Color-normal: original D-type color-weak: 2D compensation

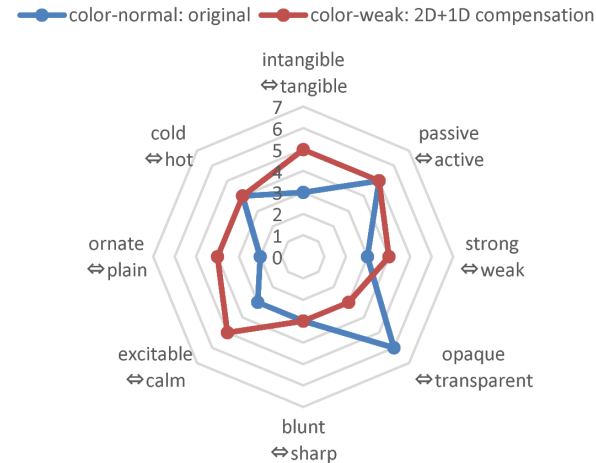

Fig. 21: "Pond"

Color-normal: original D-type color-weak: 2D+1D compensation

is due to the gamut shrinking in the higher lightness level.

The average $L^{*}$ value is larger in 2D+1D compensation than in $3 \mathrm{D}$ compensation, which is because the former expands the lightness the same rate for every $u^{*} v^{*}$ which could be too strong, but the latter takes into account of properties for each chromaticity. It is therefore more flexible. In fact, it can be observed that the $2 \mathrm{D}$ compensation enhanced both

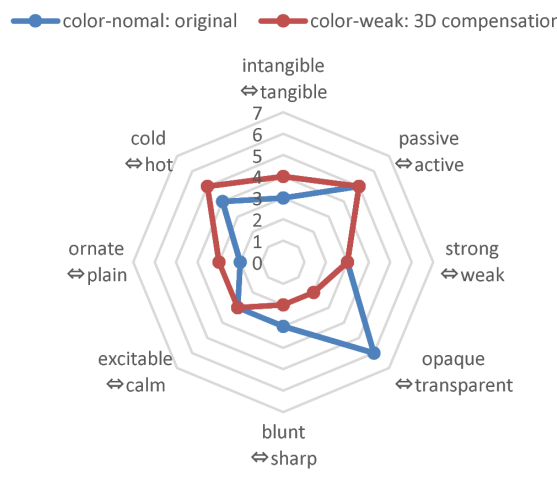

Fig. 22: "Pond"

Color-normal: original D-type color-weak: 3D compensation
TABLE VI: Averages of CIELUV values

\begin{tabular}{ll|ccc} 
Image & Algorithm & $L^{*}$ & $u^{*}$ & $v^{*}$ \\
\hline "Lake" & original & 22.8666 & 13.0871 & 38.3313 \\
& 2D & 22.5367 & 22.1902 & 38.6488 \\
& 2D+1D & 27.5266 & 12.9285 & 29.9181 \\
"Pond" & 3D & 25.0195 & 16.0550 & 33.2527 \\
& original & 19.3940 & 24.6891 & 32.6535 \\
& 2D & 18.2399 & 36.9779 & 31.9029 \\
& 2D+1D & 22.8467 & 21.6778 & 29.3835 \\
& 3D & 20.3572 & 30.2214 & 27.6951
\end{tabular}

red and green but with quite different $L^{*}$ values. Meanwhile, the $3 \mathrm{D}$ compensation combined chromaticity with lightness to produce more natural tone and contrast and therefore a balanced color distribution.

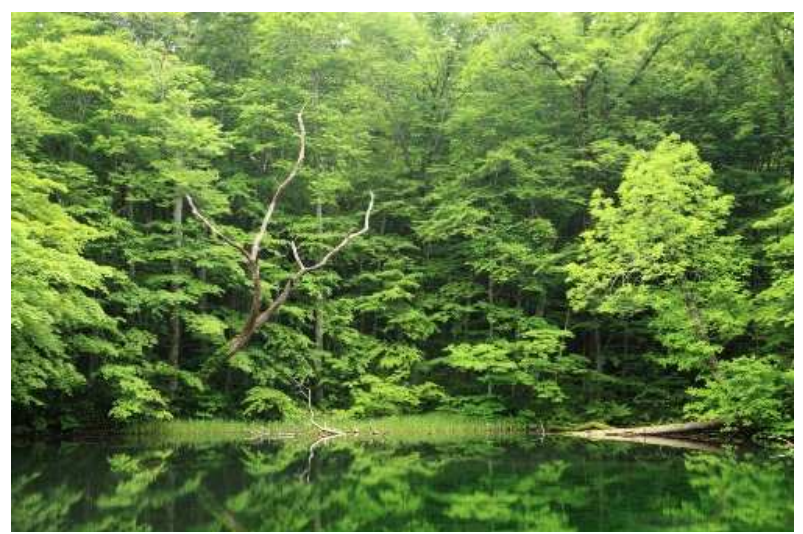

Fig. 23: "Lake": Original

\section{$X$. DisCUSSION AND SUMmary}

We described an approach to investigate the relation between the color perception of color-weak and color-normal observers. We showed that Riemann geometry provides the tools for converting image information between two different observers. The experiments showed that applying these techniques one can narrow the differences between the perceived images compared to unprocessed images. One problem one has to consider is the restrictions of the display hardware available today. It is designed for color normal observers. For additive displays such as monitors the gamut is restricted by the properties of the primary colors. One can expect that a new generation of displays may help to improve the situation.

Summarizing the main contributions of the paper we conclude that we introduced Riemann normal coordinates as a tool to construct mappings between the color spaces with metrics defined by the perception properties of different observers. These metrics were measured for color-weak and color-normal observers and 2D and 3D color-weak compensation were implemented. The semantic differential evaluation showed that both the $2 \mathrm{D}$ and the $3 \mathrm{D}$ compensations increased correlation between impressions of the color-normal and the color-weak observer and that the 3D compensation is more efficient than the $2 \mathrm{D}$ compensation. 


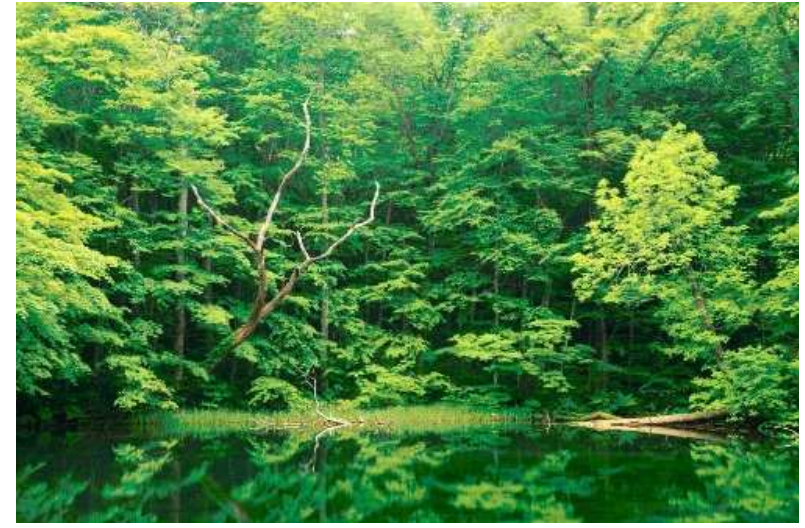

(a) "Lake": 2D compensation

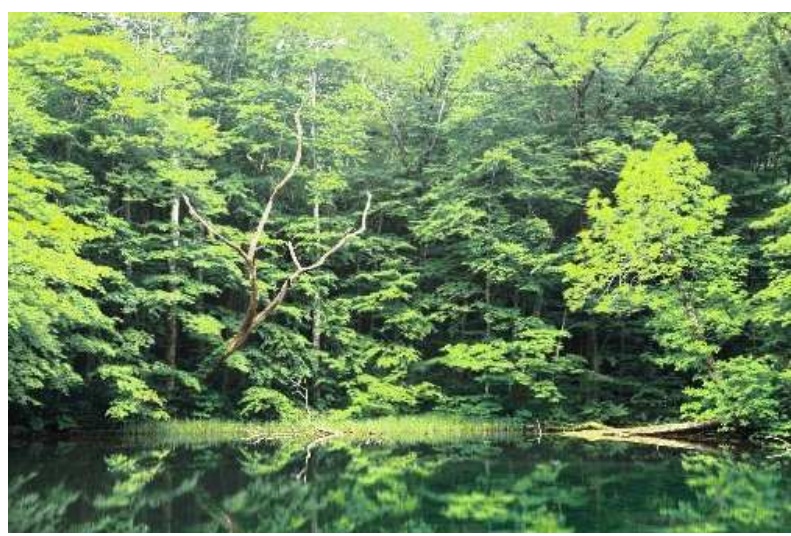

(c) "Lake": 2D+1 compensation

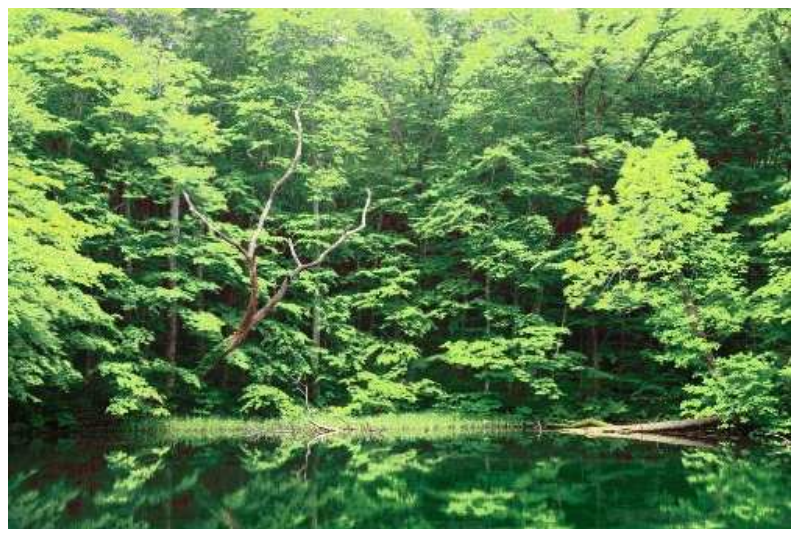

(e) "Lake": 3D compensation

\section{ACKNOWLEDEMEnTS}

The authors wish to thank Prof. Miyoshi Ayama for valuable suggestions on experiments. This work was supported by Japan Society for the Promotion of Science (JSPS) KAKENHI Grant Number 23500156 and Institute of Science and Engineering, Chuo University. The support of the Swedish Research Council through a framework grant for the project Energy Minimization for Computational Cameras (2014-6227) and by the Swedish Foundation for Strategic Research through grant IIS11-0081 is gratefully acknowledged.

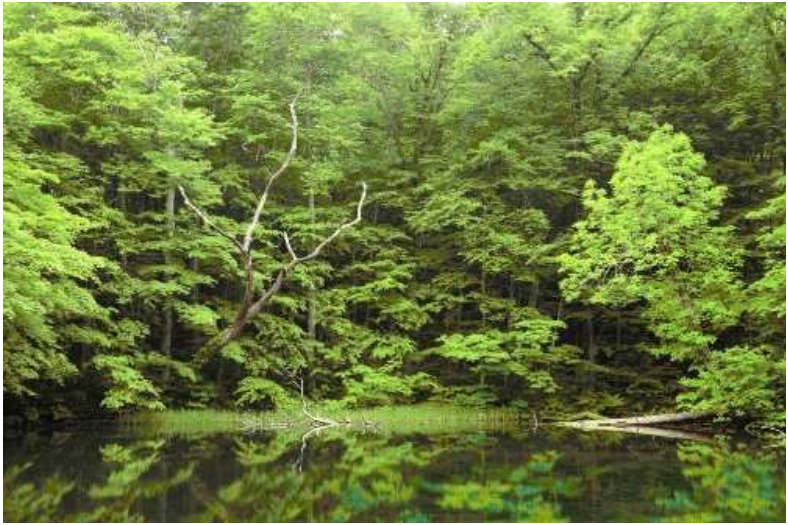

(b) "Lake": 2D simulation

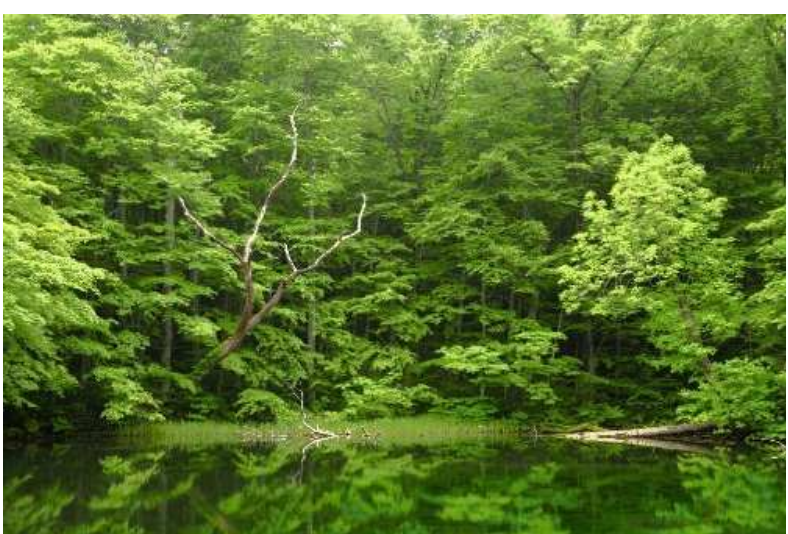

(d) "Lake": 2D+1 simulation

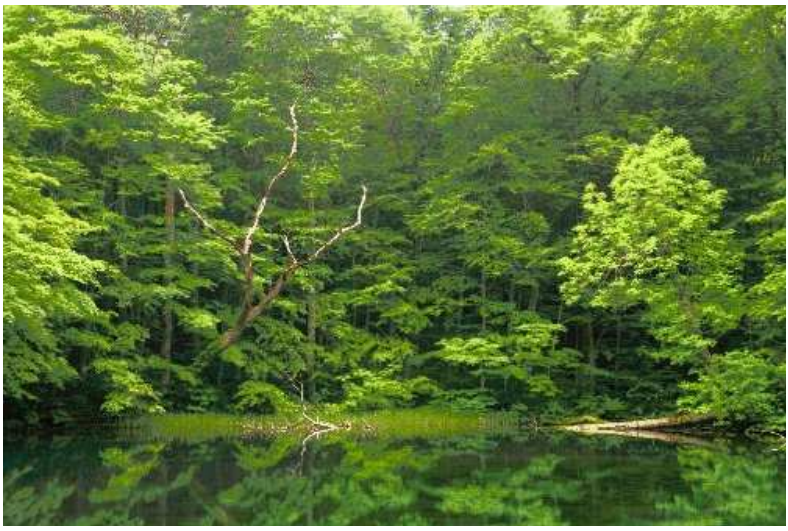

(f) "Lake": 3D simulation

\section{REFERENCES}

[1] E. Goldstein, Sensation and perception. Cengage Learning, 2013.

[2] J. Koenderink, Color for the Sciences. MIT Press, 2010.

[3] R. G. Kuehni, Color space and its divisions : color order from antiquity to the present. Hoboken, NJ: John Wiley \& Sons Inc., 2003.

[4] G. Wyszecki and W. S. Stiles, Color science : concepts and methods, quantitative data and formulae, 2nd ed. New York: Wiley, 1984.

[5] T. Jetsu, "Modeling color vision," Ph.D. dissertation, University of Eastern Finland, 2010

[6] G. M. Machado, M. M. Oliveira, and L. A. Fernandes, "A physiologically-based model for simulation of color vision deficiency," Visualization and Computer Graphics, IEEE Transactions on, vol. 15, no. 6, pp. 1291-1298, 2009.

[7] P. Olsson, O. Lind, and A. Kelber, "Bird colour vision: behavioural 


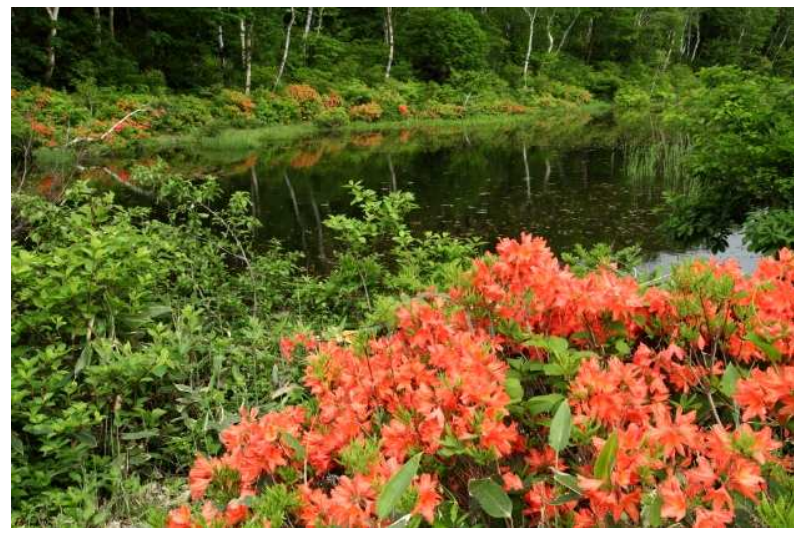

Fig. 24: "Pond": Original

thresholds reveal receptor noise," The Journal of experimental biology, vol. 218, no. 2, pp. 184-193, 2015.

[8] R. Mochizuki, T. Nakamura, J. Chao, and R. Lenz, "Color-weak correction by discrimination threshold matching," in Conference on Colour in Graphics, Imaging, and Vision. IS\&T, 2008, pp. 208-213.

[9] R. Mochizuki, S. Oshima, R. Lenz, and J. Chao, "Exact compensation of color-weakness with discrimination threshold matching," in Universal Access in Human-Computer Interaction. Applications and Services. Springer, 2011, pp. 155-164.

[10] R. Mochizuki, S. Oshima, and J. Chao, "Fast color-weakness compensation with discrimination threshold matching," in Computational Color Imaging. Springer, 2011, pp. 176-187.

[11] Y. Chen, Y. Takahashi, Y. Guan, T. Ishikawa, H. Eto, T. Nakatsue, J. Chao, and M. Ayama, "Kansei evaluation of color images corrected for color anomalies assessed by deuteranomalous and normal observers," in Proc. 17th Int. Display Workshop, 2010.

[12] Y.-C. Chen, Y. Guan, T. Ishikawa, H. Eto, T. Nakatsue, J. Chao, and M. Ayama, "Preference for color-enhanced images assessed by color deficiencies," Color Research \& Application, vol. 39, no. 3, pp. 234251, 2014.

[13] P. Petersen, Riemannian geometry. Springer, New York, 2006.

[14] S. Ohshima, R. Mochizuki, J. Chao, and R. Lenz, "Color reproduction using Riemann normal coordinates," in Computational Color Imaging. Springer, 2009, pp. 140-149.

[15] C. E. Osgood, G. J. Suci, and P. H. Tannenbaum, The measurement of meaning. Urbana: University of Illinois Press, 1957.

[16] M. do Carmo, Riemannian Geometry. Birkhäuser Boston, 2013.

[17] W. Brown and D. MacAdam, "Visual sensitivities to combined chromaticity and luminance differences," JOSA, vol. 39, no. 10, pp. 808-823, 1949.

[18] R. Mochizuki, T. Kojima, R. Lenz, and J. Chao, "Color weak compensation using local affine isometry based on discrimination threshold matching," JOSAA, vol. 32, no. 11, pp. 2093-2103, 2015.

[19] J. Chao, I. Osugi, and M. Suzuki, "On definitions and construction of uniform color space," in Conference on Colour in Graphics, Imaging, and Vision. IS\&T, 2004, pp. 55-60.

[20] J. Chao, R. Lenz, D. Matsumoto, and T. Nakamura, "Riemann geometry for color characterization and mapping," in Conference on Colour in Graphics, Imaging, and Vision, no. 1. IS\&T, 2008, pp. 277-282.

[21] H. Brettel, F. Vienot, and J. Mollon, "Computerized simulation of color appearance for dichromats," JOSAA, vol. 14, no. 10, pp. 2647-2655, 1997.

[22] R. Lenz, S. Oshima, R. Mochizuki, and J. Chao, "An invariant metric on the manifold of second order moments," in 12th Int. Conf. Computer Vision Workshops. IEEE, 2009, pp. 1923-1930.

[23] R. Lenz, R. Mochizuki, and J. Chao, "Iwasawa decomposition and computational Riemannian geometry," in 20th International Conference on Pattern Recognition. IEEE, 2010, pp. 4472-4475.

[24] B. Regan, J. Reffin, and J. Mollon, "Luminance noise and the rapid determination of discrimination ellipses in colour deficiency," Vision research, vol. 34, no. 10, pp. 1279-1299, 1994.

[25] H. Akima, "Algorithm 433: interpolation and smooth curve fitting based on local procedures [e2]," Communications of the ACM, vol. 15, no. 10, pp. 914-918, 1972.
Satoshi Oshima Satoshi Oshima received his B.E. M.E. and Ph.D degrees in Information and System Engineering from Chuo University in 2008, 2010 and 2014. His main research subject is on compensation of color-weak vision.

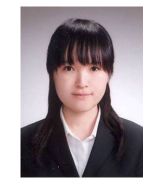

Rika Mochizuki Rika Mochizuki received her B.E., M.E. and Ph.D. degrees in Engineering from Chuo University in 2007, 2009 and 2011, respectively. She joined the Nippon Telegraph and Telephone Corporation (NTT) in 2009. She is a visiting researcher at the Chuo University from 2009. She is now a researcher in NTT Service Evolution Labs. Her research interests include color vision compensation, Life-log processing and their applications for communication support.

Reiner Lenz Reiner Lenz received his diploma degree in mathematics from the Georg August Universität in Göttingen, Germany and the $\mathrm{PhD}$. degree in Computer Engineering from Linköping University, Sweden. Before moving to Sweden he was a research assistant at Stuttgart University, Germany. His current research interests include group theoretical methods in signal processing, extreme value statistics and color signal processing.

Jinhui Chao Jinhui Chao received his B.E. degree in EE Eng from Xidian University, China 1982, M.E. and Dr. E. degrees from Tokyo Institute of Technology, Japan 1985 and 1988. He became an Assistant Professor in Tokyo Institute of Technology 1989, an Associate Professor and a Professor in Chuo University 1992 and 1996. His current research interests include Riemann geometric modeling of visual and color perception, manifold learning and cryptography theory. He is a member of IEEE and a Fellow of IEICE Japan. 


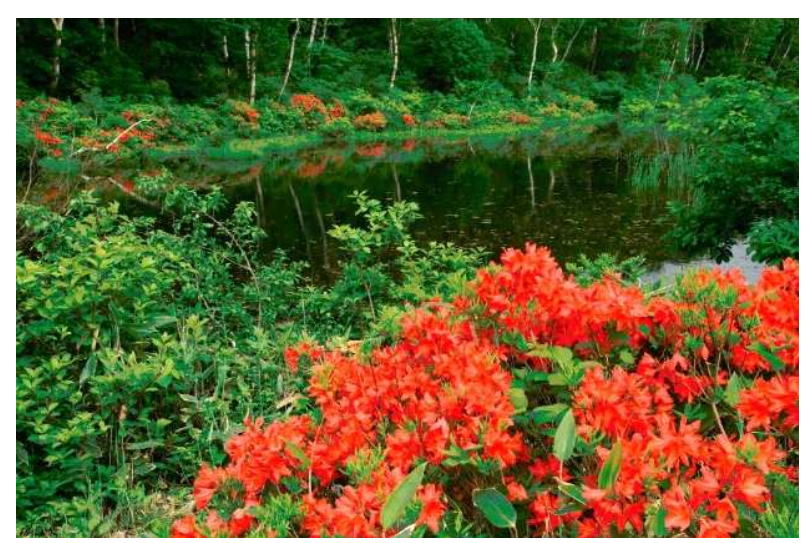

(a) "Pond": 2D compensation

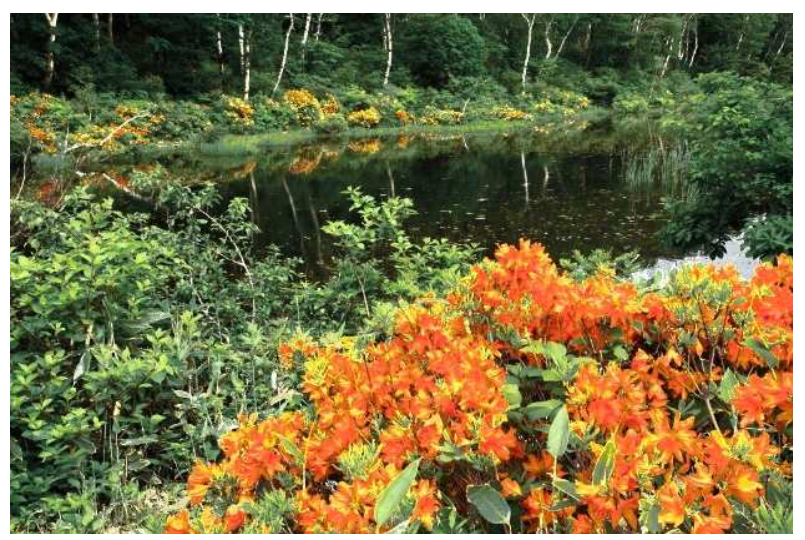

(c) "Pond": 2D+1 compensation

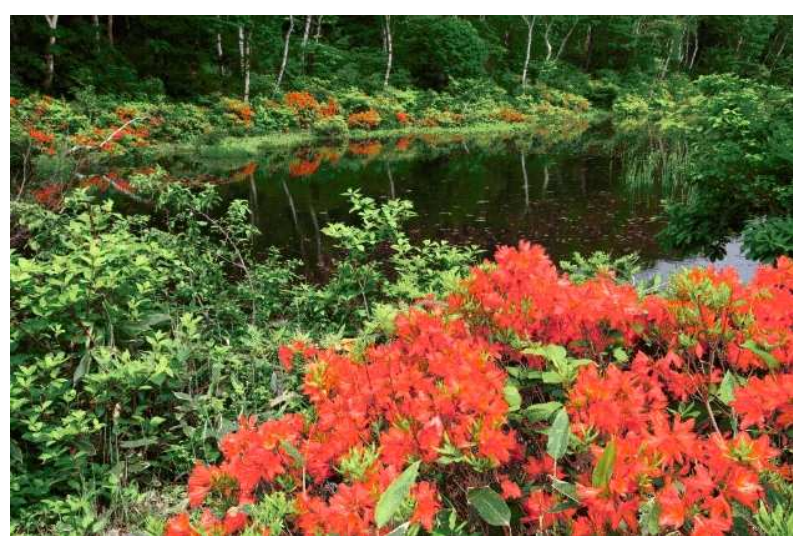

(e) "Pond": 3D compensation

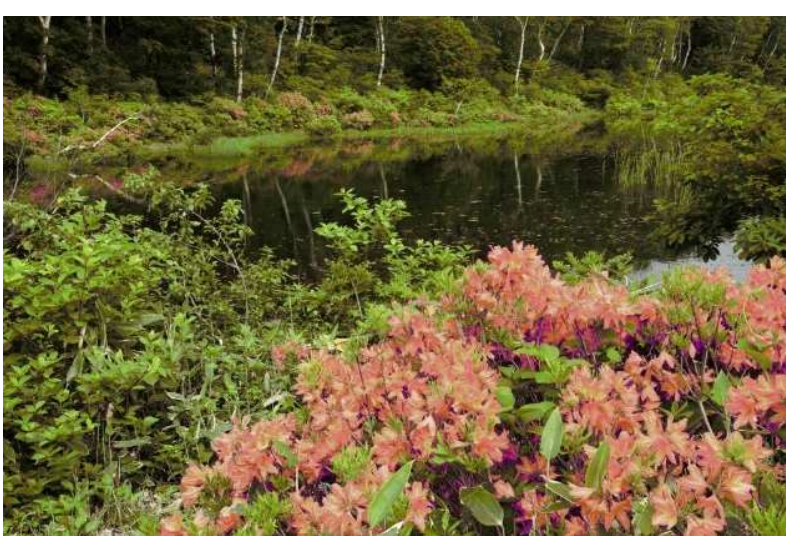

(b) "Pond": 2D simulation

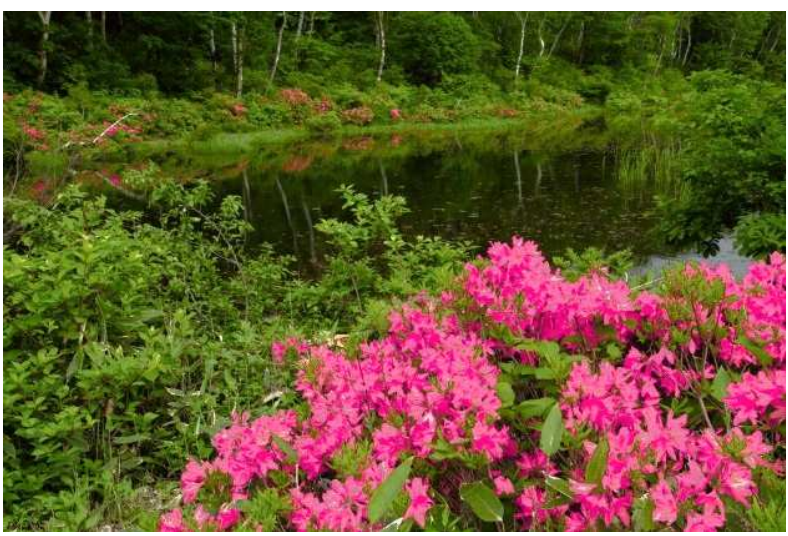

(d) "Pond": 2D+1 simulation

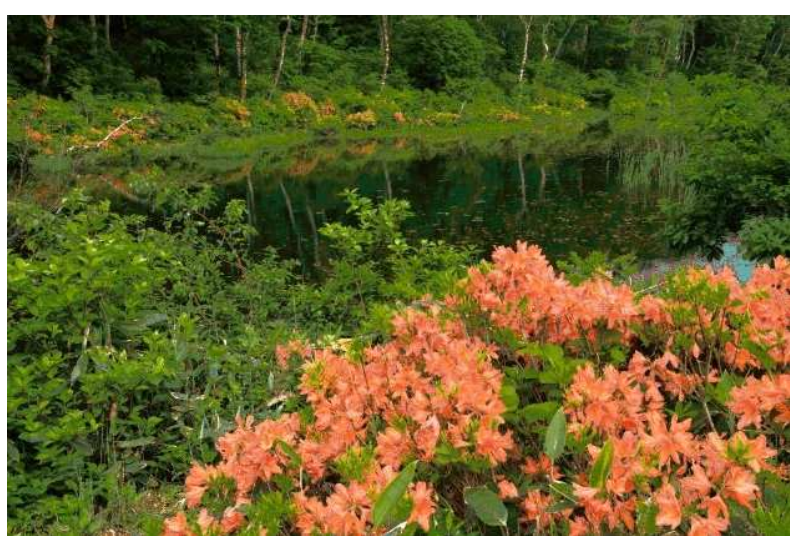

(f) "Pond": 3D simulation 